\title{
Pirolisis de plásticos de invernadero para recuperar ceras líquidas útiles para refinación
}

\author{
Paúl Palmay \\ paul.palmay@espoch.edu.ec \\ Escuela Superior Politécnica de Chimborazo - \\ Escuela de Ingeniería Química, Riobamba \\ Grupo de investigación y desarrollo GIADE Riobamba-Ecuador \\ Carlos Medina \\ carlos.medinas@espoch.edu.ec \\ Escuela Superior Politécnica de Chimborazo - \\ Escuela de Ingeniería Química, Riobamba \\ Grupo de investigación y desarrollo GIADE Riobamba-Ecuador \\ Katherine Vargas \\ katherine.vargas@espoch.edu.ec \\ Grupo de investigación y desarrollo GIADE Riobamba-Ecuador
}

\section{RESUMEN}

Esta investigación tuvo como objetivo estudiar las condiciones óptimas de pirólisis usando plástico de invernadero (LDPE) residual para obtener la mayor cantidad de ceras líquida. Para cada ensayo $100 \mathrm{~g}$ de plástico se alimentaron a un reactor batch con atmósfera inerte de nitrógeno y un sistema de refrigeración con agua $\left(10^{\circ} \mathrm{C}\right)$ para la recolección de condensables. Para evaluar el efecto de la temperatura en el rendimiento del proceso se realizaron pruebas a cinco temperaturas entre $350-450^{\circ} \mathrm{C}$, con una tasa de calentamiento de $13^{\circ} \mathrm{C} / \mathrm{min}$. A la fracción liquida obtenida se caracterizó por cromatografía de gases y se determinó propiedades como: gravedad API, punto de inflamación, poder calórico y contenido de azufre. El producto de pirólisis fue una cera oleosa compuesta por parafinas, naftenos y olefinas, de alto poder calorífico (46.49 $\mathrm{MJ} / \mathrm{Kg}$ ), relativamente limpia, capaz de ser utilizada para obtener combustibles refinados. La temperatura que genera mayor rendimiento $(67.85 \%)$ de productos líquidos es de $400^{\circ} \mathrm{C}$, con un tiempo de residencia de $6 \mathrm{~min}$. Por lo que, se concluye que la pirólisis de LDPE genera una mezcla rica de hidrocarburos alifáticos (93.52\%) a esa temperatura; mientras que temperaturas mas altas se favorece la formación de gases no condensables y ceras pesadas.

Palabras clave: Pirólisis térmica; LDPE; hidrocarburos; plástico de invernadero. 


\title{
Liquid waxes recovery from greenhouse plastics pyrolysis useful for refining
}

\begin{abstract}
The objective of this research was to study the optimal pyrolysis conditions using residual greenhouse plastic (LDPE) to generate the highest amount of liquid waxes. $100 \mathrm{~g}$ of plastic were fed to a batch reactor with an inert nitrogen atmosphere and a cooling system (water at $10^{\circ} \mathrm{C}$ ) was used to collect condensable fraction in each test. The effect of temperature on performance of the process were evaluated at five temperatures from 350 to $450^{\circ} \mathrm{C}$, with $13^{\circ} \mathrm{C} / \mathrm{min}$ of heating rate. The liquid fraction obtained was characterized by gas chromatography and the properties such as: API gravity, flash point, caloric power and sulfur content were determined. The pyrolysis product was a relatively clean oily wax composed of paraffins, naphthenes and olefins with high calorific power (46.49 MJ / Kg), capable to be used in fuel refining. The temperature that generates the highest yield $(67.85 \%)$ of liquid products is $400^{\circ} \mathrm{C}$, with $6 \mathrm{~min}$ of residence. Therefore, we concluded that pyrolysis of LDPE generates a mixture of aliphatic hydrocarbons (93.52\%) at that temperature; while higher temperatures conduce to non-condensable gases and heavy waxes.
\end{abstract}

Keywords: Thermal pyrolysis; LDPE; hydrocarbons; greenhouse plastic.

Artículo recibido: 05 de Abril 2021 Aceptado para publicación: 28 de Mayo 2021 Correspondencia: carlos.medinas@espoch.edu.ec Conflictos de Interés: Ninguna que declarar 


\section{INTRODUCTION}

La producción agrícola es una actividad importante de nuestro país, por tal razón es una rama en desarrollo tecnológico para mejorar la productividad. En base a ello, los plásticos se han convertido en materiales impresindibles en la construcción de invernaderos sobre todo en zonas de alta producción agrícola de Ecuador, pero tambien se han introducido al mercado para suplir a materiales como vidrio, metales, caucho o madera. En la mayoría de los casos, los productos generados a base de plástico se producen industrialmente a partir de derivados del petróleo que se componen de hidrocarburos, además incluyen un conjunto de agentes aditivos que buscan mejorar las propiedades de los plásticos, estos aditivos pueden ser antioxidantes, colorantes o estabilizadores que no siempre son deseables para el medio ambiente (Brems, Baeyens, \& Dewil, 2012)

La demanda de plásticos a nivel mundial se encuentra en constante crecimiento, por lo tanto, la generación de residuos de este tipo también se incrementa. En el año 2018 la producción global de plásticos alcanzó un total de 359 millones de toneladas, con un incremento anual del 3.16\%. De este total, unos 4.6 millones de toneladas de plásticos se utilizan anualmente en aplicaciones agrícolas en todo el mundo (Plastics Europe \& Conversio Market \& Strategy GmbH, 2019). De los cuales, 3.5 millones de toneladas son películas de invernadero, cada una de las cuales representa más de 1.0 millón de toneladas / año en desechos (Li et al., 2020). En el caso de Ecuador, la producción de flores, frutas y hortalizas con técnicas de invernadero va en aumento año tras año, lo cual exige el uso de plásticos de invernadero. La provincia de Pichincha, la principal productora florícola, cuenta con aproximadamente 850 ha de cultivo de flores, donde se emplean alrededor de 2000 kg de plástico por cada hectárea (Terán, 2010).

De manera general los plásticos se puden obtener de varios precursores, gracias a la alta rentabilidad y facilidad de aplicación, así pues, en la fabricación de plásticos a nivel global se usa polietileno en un $32 \%$, el polipropileno en un $20 \%$, seguido del policloruro de vinilo (PVC) con el 17\% (Li et al., 2020). De este manera, los desechos plásticos de invernadero representan un considerable cumulo de bienes, que luego de haberse usado, son desechados de forma inadecuada y solo tienen un tiempo de vida útil de aproximadamente 2 años (Anuar Sharuddin, Abnisa, Wan Daud, \& Aroua, 2016). Los desechos plásticos se constituyen por tanto de polietileno de alta y baja densidad, polipropileno, poliestireno y tereftalato de polietileno. Solo el $2 \%$ de los desechos son 
reincorporados como materia prima de reciclado en la producción de nuevos materiales de plástico (D'ambrières, 2019), del 65 al 70\% se disponen en vertederos, el 14\% de ellos se utilizan en la recuperación de energía y el resto se incineran (Zhang et al., 2018). Tanto los vertederos como la incineración son costosos y desfavorables por diferentes razones, que incluyen, entre otras, afecciones ambientales por la alta emision de gases de efecto invernadero (Miskolczi, Angyal, Bartha, \& Valkai, 2009).

Los plásticos de invernadero comunmente se elaboran a base de polietileno de baja densidad (LDPE) debido a su bajo costo, el cual se producen mediante polimerización por adición del etileno utilizando catalizadores organometálicos que generan productos con un alto grado de ramificación de cadena corta y larga (Makhlouf, Hassan, Nour, Abdelmonem, \& Abdelkhalik, 2017). El LDPE debido a la radiación UV y el oxígeno presenta degradación cuando esta expuesto a la intemperie, esto genera la pérdida de sus propiedades mecánicas, por lo cual, su tiempo de vida útil es bajo (Ranjan \& Goel, 2019). Además de las tecnologías de reciclaje mecánico, también existen rutas químicas para reciclar los residuos plásticos $(11,12,13)$. La pirólisis, una de las tecnologías más importantes, es la fragmnetación de materiales orgánicos (polímeros) bajo el efecto del calor, en medio inerte y al vacío (ausencia de oxígeno), utilizando agentes reductores y oxidantes, con o sin catalizador a alta temperatura $(3,11,12,14)$. Durante la fragmentación, las cadenas de polímeros se rompen y se crean nuevas moléculas. Debido a que la mayoría de estas moléculas creadas tienen carácter radical, se convierten en productos gaseosos, líquidos y sólidos al involucrarse en una serie de reacciones entre ellas.

Dependiendo de las condiciones del proceso, típicamente se obtienen como producto mayoritario una mezcla de moléculas en estado líquido que se pueden convertir en combustibles $(13,15,16)$. Además de los productos líquidos y gaseosos, la fracción sólida (residuos) que permanece en el reactor después de la pirólisis puede ser utilizada para obtener carbon activado por medio de tratamientos químicos y físicos adecuados. La descomposición térmica de residuos plásticos por pirólisis tiene ciertas ventajas sobre otras formas de tratamiento de residuos, ya que la mayoría de sus productos pueden utilizarse para destilar gasoil con una composición similar a los combustibles convencionales (Zheng et al., 2018). En la pirólisis de desechos de LDPE, por ejemplo, a $480^{\circ} \mathrm{C}$, el rendimiento total de gas y líquido alcanza hasta el $65 \%(12,13)$. Las 
condiciones de pirólisis y el tipo de material pirolizado tienen influencia directa sobre el producto de combustión obtenido y sus propiedades. La velocidad de calentamiento, la temperatura de reacción, la presión de trabajo y el tiempo de retención, son factores de pirólisis que juegan un papel importante en la estructura y cantidad de producto carbonizado (Paethanom \& Yoshikawa, 2012).

El presente estudio tuvo como objetivo establecer las variables óptimas de proceso para obtener la mayor cantidad de fracción líquida a partir de residuos de plásticos de invernadero recolectados en zonas agrícolas de la provincia de Chimborazo. Para finalmente realizar la caracterización físico-química los productos líquidos obtenidos.

\section{MATERIALES Y MÉTODOS}

\subsection{Caracterización de la Materia prima}

Se utilizó $1 \mathrm{~kg}$ de residuos de plástico de invernadero proveniente de la zona de Tunshi provincia de Chimborazo, muestra que previamente fue lavada, secada y cortada en pequeñas láminas de aproximadamente $1.5 \mathrm{~cm}$. La caracterización se realizó mediante prueba de solubilidad para identificación de plásticos, empleando: agua destilada, alcohol isopropílico al $40 \%$ y aceite de maíz. Se realizó tambien un análisis de espectroscopía infrarroja por transformada de Fourier (espectrómetro JASCO FT/IR-4100). Los espectros IR de 4000 a $550 \mathrm{~cm}^{-1}$ fueron tomados y procesados utilizando el programa Spectra Analysis, que sirvió para la identificación de la estructura del film.

\subsection{Pirólisis térmica}

Se usó un reactor tipo batch, con un sistema de válvulas que ayudan a la inyección de nitrógeno y la recolección de condensables; salida que está conectada a un sistema de refrigeración que utiliza agua a $10{ }^{\circ} \mathrm{C}$. La pirólisis de las películas de plástico de invernadero se llevó a cabo a temperaturas de $350,375,400,425$ y $450^{\circ} \mathrm{C}$ con tres repeticiones cada una. Cada ensayo trabajó con lotes de $100 \mathrm{~g}$ de materia prima a una tasa de calentamiento de $15^{\circ} \mathrm{C} \min ^{-1}$ y un tiempo de permanencia de 20 minutos.

\subsection{Caracterización de la fracción líquida}

El producto principal de la pirólisis, se analizó en detalle mediante Cromatografía de Gases acoplada a Espectrometría de Masas (GC/MS), para determinar su contenido de compuestos alifáticos y aromáticos. Las propiedades físicoquímicas que se determinaron son: densidad, viscosidad, poder calórico, punto de inflamación, cenizas y contenido de azufre. La densidad específica se determinó mediante el uso de un picnómetro de $50 \mathrm{ml}$ 
y una balanza analítica A\&D HR-250A. Para determinar la viscosidad cinemática, se siguió el Método Estándar D445 de la American Society for Testing and Materials (ASTM), para lo cual, se empleó un viscosímetro calibrado y un sistema de calentamiento. La medición del poder calórico se basó en la Norma ASTM D240 "Determinación del calor de combustión de hidrocarburos" mediante el uso de una bomba calorimétrica con electrodos de ferroníquel y un sistema de control de temperatura. Mientras que, para evaluar el punto de inflamación se desarrolló un Método de Prueba basado en la Norma ASTM D56 "Determinación del punto de inflamación” por medio del uso de un analizador manual Tag de vaso cerrado que indicó la temperatura flash point. La cantidad de ceniza se halló siguiendo los lineamientos de la Norma ASTM D482 “Determinación de cenizas en productos del petróleo", siendo para ello necesario, una mufla PLATA06. Para la determinación del contenido de azufre se aplicó la norma ASTM4294-16e1 "Determinación de azufre en petróleo y productos derivados del petróleo", para lo cual, fue requerido un Analizador de rayos-X.

\section{RESULTADOS Y DISCUSIÓN}

\subsection{Espectroscopía Infrarroja FT-IR de materia prima}

En la figura 1 se puede observar el espectro IR del polietileno analizado donde se puede observar movimientos de tensión de los enlaces C-H alrededor de $2900 \mathrm{~cm}^{-1}$ y $2800 \mathrm{~cm}^{-}$ ${ }^{1}$, deformación del grupo $-\mathrm{CH}_{2}$ cerca de $1465 \mathrm{~cm}^{-1}$ y por la zona de $700 \mathrm{~cm}^{-1}$ vibraciones de flexión de los enlaces C-C (TORRES MORENO \& CASTAÑEDA SÁENZ, 2018). Y especialmente una banda débil a $\sim 1378 \mathrm{~cm}^{-1}$ propia del polietileno de baja densidad (Crespo, 2013). Adicional, un pico a $2350 \mathrm{~cm}^{-1}$ correspondiente al enlace aislado $\mathrm{C} \equiv \mathrm{N}$, este último indica la presencia de aditivos nitrogenados como el acrilonitrilo o aminas impedidas HALS, empleados como estabilizantes y antioxidantes utilizados en el tratamiento del plástico (DELGADO, 2019).

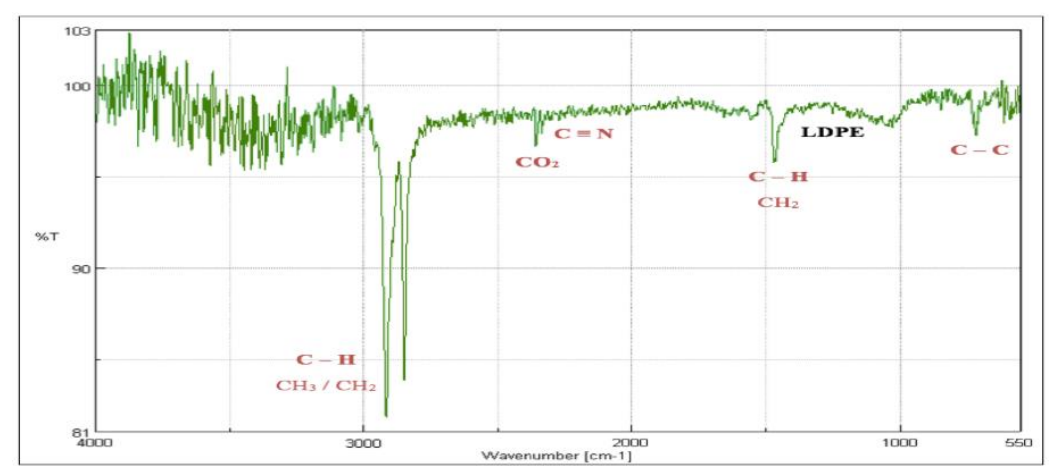

Figura 1. Espectro IR de películas plásticas de invernadero. 


\subsection{Prueba de densidad de materia prima}

La prueba de densidad demostró que el termoplástico es de tipo LDPE (Polietileno de Baja Densidad), cuyo valor de densidad oscila entre $0.917-0.925 \mathrm{~g} / \mathrm{cm}^{3}$ (Suastes, No, Mar, Tláhuac, \& México, 2018); ya que, al sumergirlo en tres medios, como se observa en la Figura 2 y por diferencia de densidades, revela que se trata de una poliolefina (LDPE, HDPE, PP) con densidad comprendida entre $0.930-0.910 \mathrm{~g} / \mathrm{cm}^{3}$, que la clasifica como LDPE (Vitz et al., 2019).

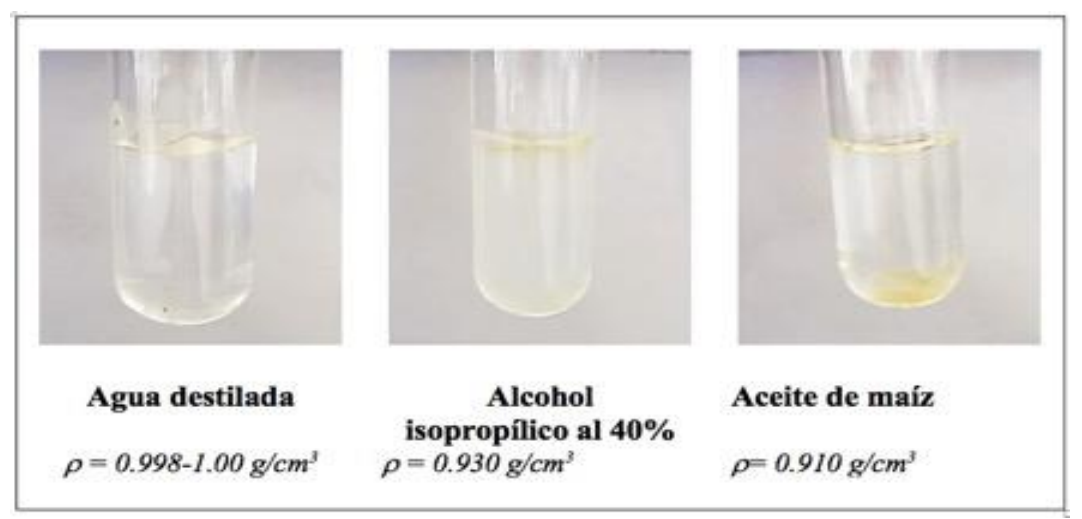

Figura 2. Prueba de densidad del plástico de invernadero.

\subsection{Contenido de humedad de materia prima}

El porcentaje de humedad fue de $0.01 \%$, cuyo valor resulta conveniente para el correcto desarrollo de la pirólisis, ya que, favorece la creación de la atmósfera inerte dentro del reactor, evita el gasto de energía que se requeriría para eliminar el contenido de agua y permite un mejor aprovechamiento del poder calorífico del plástico (DELGADO, 2019)

\subsection{Proceso de pirólisis}

La Tabla 1 muestra el rendimiento de los productos derivados de la pirólisis de LDPE proveniente de residuos de plástico de invernadero. El mayor rendimiento para la fracción líquida se alcanza a $\operatorname{los} 400{ }^{\circ} \mathrm{C}$, con un producto semilíquido aceitoso que al llegar a la temperatura ambiente se solidifica, pasando a ser un material ceroso de baja viscosidad, color café pardo brillante y olor ocre.

Tabla 1. Rendimiento de los productos finales de la pirólisis térmica de LDPE.

\begin{tabular}{lccccc}
\hline \multirow{2}{*}{$\begin{array}{c}\text { Fracción } \\
(\%)\end{array}$} & $\mathbf{3 5 0}$ & $\mathbf{3 7 5}$ & $\mathbf{4 0 0}$ & $\mathbf{4 2 5}$ & $\mathbf{4 5 0}$ \\
\cline { 2 - 6 } & 11.85 & 5.85 & 3.05 & 2.31 & 2.70 \\
\hline Gaseosa & 55.92 & 57.25 & 67.85 & 56.15 & 56.72 \\
Líquida & 32.22 & 36.89 & 29.09 & 41.53 & 40.57 \\
Sólida & &
\end{tabular}


A medida que la temperatura de pirólisis se incrementa, existe un marcado aumento del rendimiento de gases, de $11.85 \%$ en peso a $350^{\circ} \mathrm{C}$ a más de $40 \%$ en peso a $450^{\circ} \mathrm{C}$, con una disminución considerable de la fracción aceite/cera. El carbón correspondiente a la fracción sólida, por su parte disminuye su rendimiento con el aumento de temperatura pasando de $32.22 \%$ en peso a $350^{\circ} \mathrm{C}$ a $2.70 \%$ en peso a $450^{\circ} \mathrm{C}$. Para todas las condiciones de temperatura establecidas, la fracción líquida al final mostró consistencia cerosa como se observa en la Figura 3, su diferencia radicó en el color y textura. A 350 y $375^{\circ} \mathrm{C}$ resulta amarillenta y semisólida, mientras que, a 425 y $450{ }^{\circ} \mathrm{C}$ es más oscura y altamente sólida.

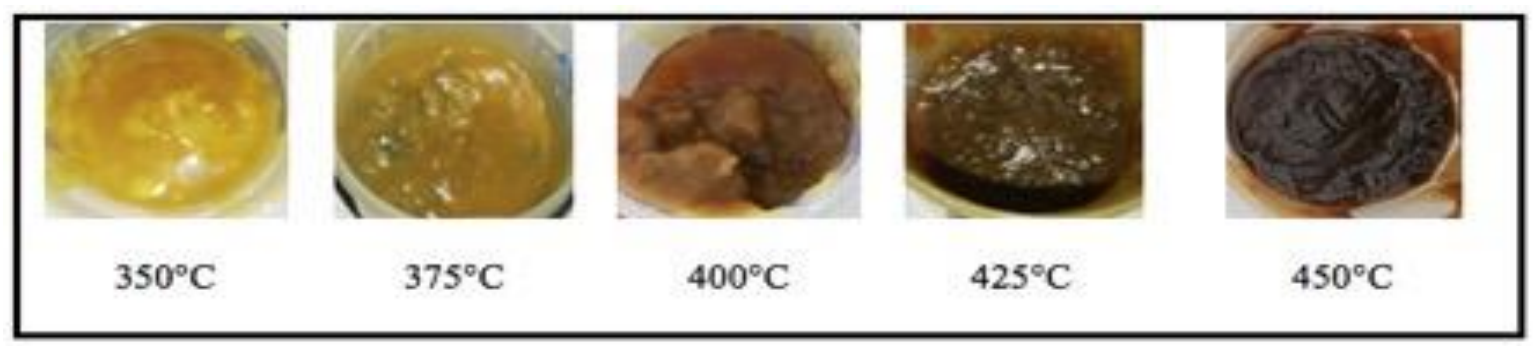

Figura 3. Fracción líquida a las diferentes temperaturas de pirólisis.

La Figura 4 muestra la variación de temperatura frente al tiempo que tarda el proceso de pirólisis. Durante los primeros $18 \mathrm{~min}$ se incrementa de manera uniforme hasta los $340^{\circ} \mathrm{C}$, donde decae levemente y luego empieza a estabilizase, para cada una de las condiciones establecidas. Esta condición se debe a que en este punto inicia la degradación térmica del plástico y termina aproximadamente a $\operatorname{los} 440^{\circ} \mathrm{C}$ alrededor de los 35 min. Estos resultados, coinciden con investigaciones previas, donde se indica que la degradación térmica del LDPE ocurre entre los 360 a $410^{\circ} \mathrm{C}$ (Hartulistiyoso, Sigiro, \& Yulianto, 2015) o entre los 375 a $450^{\circ} \mathrm{C}$.

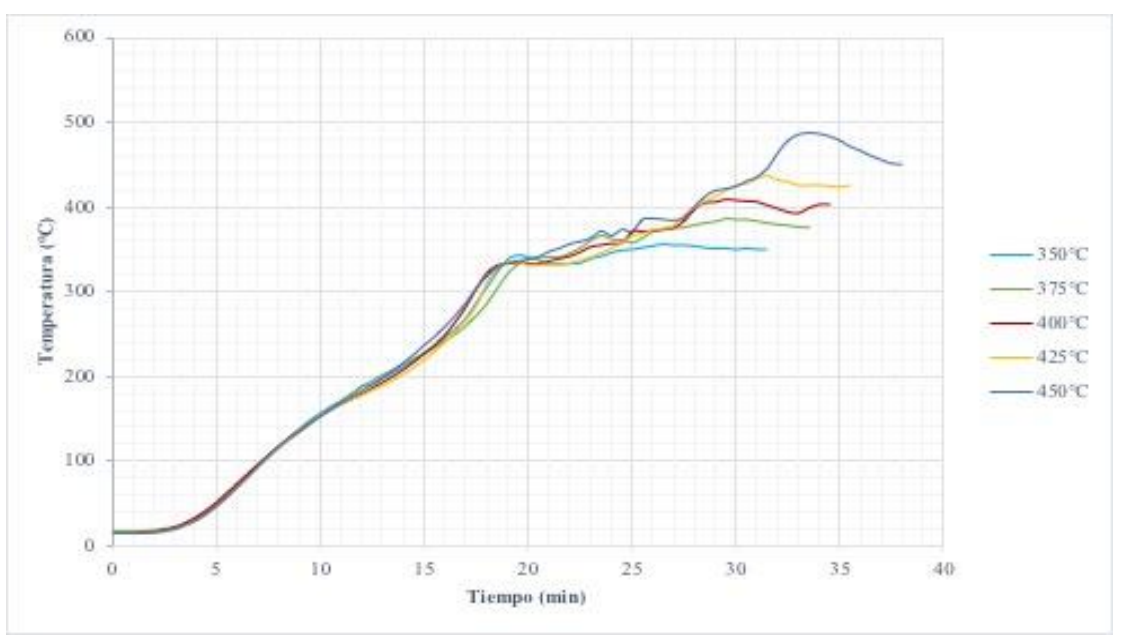

Figura 4. Perfil de temperatura de la pirólisis de LDPE. 


\subsection{Análisis cromatográfico}

El análisis cromatográfico identificó 43 compuestos que se los clasifica en seis grupos: alcanos, alquenos, cetenas, alcoholes, aminas y ácidos carboxílicos. Donde los alcanos lideraron la lista con un $53.74 \%$, seguidos de los alquenos con un $39.78 \%$ y las cetenas con un $4.23 \%$, principalmente. Dando un total de $93.52 \%$ de hidrocarburos totales, por lo que, resulta ser un petroquímico base rico en parafinas, olefinas y naftenos, que puede utilizarse como materia prima dentro de la industria petroquímica. El LDPE se degradó esencialmente por escisión aleatoria debido a la presencia de cadenas laterales, favoreciendo la formación de radicales terciarios por ser más estables. En consecuencia, las reacciones de transferencia de hidrógeno dominan el proceso frente a las rupturas $\beta$ que forman el etileno. Lo que conduce a la obtención de una mezcla compleja de hidrocarburos saturados e insaturados de variado número de carbonos.

Tabla 2. Composición de la cera oleosa.

\begin{tabular}{cc}
\hline $\mathbf{N}^{\circ}$ de carbonos & Composición (\%) \\
\hline $\mathrm{C}_{6}-\mathrm{C}_{12}$ & 10.74 \\
$>\mathrm{C}_{12}-\mathrm{C}_{23}$ & 68.22 \\
$>\mathrm{C}_{23}$ & 14.56 \\
\hline Hidrocarburos Totales & 93.52
\end{tabular}

\subsection{Propiedades fisicoquímicas de la cera}

En la Tabla 2 se resumen las propiedades físico-químicas determinadas para la cera pirolítica. La densidad específica al tener un valor de $0.81 \mathrm{~g} / \mathrm{cm}^{3}$, define al producto como un hidrocarburo ligero con bajo contenido de nitrógeno y azufre, constituido principalmente por alcanos lineales o ramificados y ciclo alcanos (Correa Pabón \& Souza Filho, 2019). El valor de viscosidad cinemática de 1.7 cSt indica de igual manera que la cera obtenida es rica en compuestos parafínicos y nafténicos. Por su parte el valor de 46.49 $\mathrm{MJ} / \mathrm{Kg}$ respecto al poder calorífico, señala que el producto tiene gran potencial energético. El punto de inflamación refleja un valor de $7.2^{\circ} \mathrm{C}$, por lo que puede arder en condiciones normales y se debe principalmente a la presencia de hidrocarburos de bajo peso molecular y al gas ceteno altamente inflamable (NJ Health, 2011). Los bajos porcentajes de ceniza y azufre sugieren la obtención de un producto limpio constituido por parafinas y olefinas aptas para utilizarse como materia prima recuperada (El-Sabagh et al., 2018). Se puede apreciar la fracción líquida como un producto rico en parafinas 
que le otorgan buenas propiedades hidrofóbicas que puede utilizarse en la fabricación de emulsiones hidrofobizantes (Bajus \& Hájeková, 2010).

Tabla 3. Caracterización físico-química de la fracción liquida (cera oleosa).

\begin{tabular}{lcc}
\multicolumn{1}{c}{ Propiedad } & Unidad & Cera pirolítica (LDPE) \\
\hline Aspecto, color y olor & - & Pasta semilíquida, pardo brillante, \\
Solubilidad en agua & - & ocre \\
& & Insoluble \\
Composición & $\%$ & 53.74: Parafinas \\
& & 49.78 : Olefinas \\
Poder calorífico & $\mathrm{MJ} / \mathrm{Kg}$ & $2.25:$ Cetenas \\
Punto de inflamación & ${ }^{\circ} \mathrm{C}$ & 46.49 \\
Densidad específica $\left(\mathbf{1 5}^{\circ} \mathbf{C}\right)$ & $\mathrm{g} / \mathrm{cm}^{3}$ & 7.2 \\
Contenido de azufre & $\%$ & 0.81 \\
Viscosidad cinemática $\left(\mathbf{1 0 0}^{\circ} \mathbf{C}\right)$ & $\mathrm{cSt}$ & 0.00194 \\
Contenido de ceniza & $\% \mathrm{p} / \mathrm{p}$ & 1.7 \\
Punto de fusión & ${ }^{\circ} \mathrm{C}$ & 0.001 \\
\hline
\end{tabular}

\section{CONCLUSIONES}

A partir de la caracterización de las películas de plástico de invernadero residuales, se determinó que está constituido mayoritariamente por polietileno de baja densidad (LDPE) y en menor proporción por aditivos nitrogenados como el acrilonitrilo y las aminas impedidas HALS, las cuales le ofrecen protección frente a la radiación UV y los agentes atmosféricos y químicos a los cuales están sometidas estas películas plásticas. La pirólisis térmica de este termoplástico, produce como producto principal una mezcla cera-aceite, carbón y gas. A medida que se incrementa la temperatura de pirólisis de 350 a $450^{\circ} \mathrm{C}$, existió una marcada disminución en la fracción liquida y un aumento de la fracción gaseosa. Por lo tanto, se estableció que las condiciones óptimas para lograr el mayor rendimiento de la fracción líquida, alrededor de $67.85 \%$, es trabajar a $400^{\circ} \mathrm{C}$ durante 6 min, con una tasa de calentamiento de $13^{\circ} \mathrm{C} / \mathrm{min}$. El análisis detallado de la cera por cromatografía GC/MS, mostró la presencia de compuestos alifáticos como parafinas, olefinas y naftenos, con una distribución del número de carbonos de C9 a C32. Constituyéndose, además, como un producto limpio da alto potencial calorífico que puede 
utilizarse en la industria petroquímica como sustitutos de materias primas derivadas del petróleo.

\section{REFERENCIAS}

Anuar Sharuddin, S. D., Abnisa, F., Wan Daud, W. M. A., \& Aroua, M. K. (2016). A review on pyrolysis of plastic wastes. Energy Conversion and Management, 115, 308-326. https://doi.org/10.1016/j.enconman.2016.02.037

Bajus, M., \& Hájeková, E. (2010). Thermal Cracking Of The Model Seven Components Mixed Plastics Into Oils/Waxes. Petroliun \& Coal, 52(3), 164-172.

Brems, A., Baeyens, J., \& Dewil, R. (2012). Recycling and recovery of post-cet alonsumer plastic solid waste in a European context. Thermal Science, 16(3), 669685. https://doi.org/10.2298/TSCI120111121B

Correa Pabón, R. E., \& Souza Filho, C. R. de. (2019). Crude oil spectral signatures and empirical models to derive API gravity. Fuel, 237(May 2018), 1119-1131. https://doi.org/10.1016/j.fuel.2018.09.098

Crespo, S. (2013). Evaluación de la pirólisis térmica de residuos de polietileno proveniente de la producción de banano en un reactor batch, 21 .

D'ambrières, W. (2019). Plastics recycling worldwide: Current overview and desirable changes. Field Actions Science Report, 2019(Special Issue), 12-21.

DELGADO, S. K. M. (2019). Obtención De Combustible Mediante Pirólisis Térmica a Partir De Polipropileno Reciclado, 145.

Dubdub, I., \& Al-Yaari, M. (2020). Pyrolysis of low density polyethylene: Kinetic study using TGA data and ANN prediction. Polymers, 12(4). https://doi.org/10.3390/POLYM12040891

El-Sabagh, S. M., Rashad, A. M., El-Naggar, A. Y., El Nady, M. M., Badr, I. A., Ebiad, M. A., \& Abdullah, E. S. (2018). API gravities, vanadium, nickel, sulfur, and their relation to gross composition: Implications for the origin and maturation of crude oils in Western Desert, Egypt. Petroleum Science and Technology, 36(1), 14211428. https://doi.org/10.1080/10916466.2017.1336774

Hartulistiyoso, E., Sigiro, F. A. P. A. G., \& Yulianto, M. (2015). Temperature Distribution of the Plastics Pyrolysis Process to Produce Fuel at 450oC. Procedia Environmental Sciences, 28(SustaiN 2014), 234-241.

https://doi.org/10.1016/j.proenv.2015.07.030 
Kane, S. N., Mishra, A., \& Dutta, A. K. (2016). Preface: International Conference on Recent Trends in Physics (ICRTP 2016). Journal of Physics: Conference Series, 755(1). https://doi.org/10.1088/1742-6596/755/1/011001

Li, Z., Zhong, Z., Zhang, B., Wang, W., Seufitelli, G. V. S., \& Resende, F. L. P. (2020). Catalytic fast co-pyrolysis of waste greenhouse plastic films and rice husk using hierarchical micro-mesoporous composite molecular sieve. Waste Management, 102, 561-568. https://doi.org/10.1016/j.wasman.2019.11.012

Makhlouf, G., Hassan, M., Nour, M., Abdelmonem, Y., \& Abdelkhalik, A. (2017). A Novel Intumescent Flame Retardant: Synthesis and Its Application for Linear Low-Density Polyethylene. Arabian Journal for Science and Engineering, 42(10), 4339-4349. https://doi.org/10.1007/s13369-017-2443-0

Miskolczi, N., Angyal, A., Bartha, L., \& Valkai, I. (2009). Fuels by pyrolysis of waste plastics from agricultural and packaging sectors in a pilot scale reactor. Fuel Processing Technology, 90(7-8), 1032-1040.

https://doi.org/10.1016/j.fuproc.2009.04.019

NJ Health. (2011). Derecho a Saber Hoja Informativa sobre Sustancias Peligrosas Descripción y uso. New Jersey Department of Health, 6. Retrieved from http://nj.gov/health/eoh/rtkweb/documents/fs/1594sp.pdf

Paethanom, A., \& Yoshikawa, K. (2012). Influence of pyrolysis temperature on rice husk char characteristics and its tar adsorption capability. Energies, 5(12), 49414951. https://doi.org/10.3390/en5124941

Plastics Europe, G. M. R., \& Conversio Market \& Strategy GmbH. (2019). Plastics - the Facts 2019, 14, 35. Retrieved from https://www.plasticseurope.org/en/resources/market-data

Qureshi, M. S., Oasmaa, A., Pihkola, H., Deviatkin, I., Tenhunen, A., Mannila, J., ... Laine-Ylijoki, J. (2020). Pyrolysis of plastic waste: Opportunities and challenges. Journal of Analytical and Applied Pyrolysis, 152(October 2019). https://doi.org/10.1016/j.jaap.2020.104804

Rahimi, A. R., \& Garciá, J. M. (2017). Chemical recycling of waste plastics for new materials production. Nature Reviews Chemistry, 1, 1-11. https://doi.org/10.1038/s41570-017-0046

Ranjan, V. P., \& Goel, S. (2019). Degradation of Low-Density Polyethylene Film 
Exposed to UV Radiation in Four Environments. Journal of Hazardous, Toxic, and Radioactive Waste, 23(4), 04019015. https://doi.org/10.1061/(asce)hz.21535515.0000453

Sogancioglu, M., Yel, E., \& Ahmetli, G. (2017). Pyrolysis of waste high density polyethylene (HDPE) and low density polyethylene (LDPE) plastics and production of epoxy composites with their pyrolysis chars. Journal of Cleaner Production, 165, 369-381. https://doi.org/10.1016/j.jclepro.2017.07.157

Suastes, Q., No, C. P., Mar, C. Del, Tláhuac, D., \& México, C. De. (2018). AGUA DESTILADA, (7), 1-8.

Terán, A. (2010). Diseño de los procesos de reducción de tamaño, secado y granceado de residuos plásticos provenientes de invernaderos, 23-82. Retrieved from https://biblioteca.epn.edu.ec/cgi-bin/koha/opacdetail.pl?biblionumber=10020\&query_desc $=\mathrm{kw} \% 2 \mathrm{Cwrdl} \% 3 \mathrm{~A}$ francisco quiroz

The American Chemistry Council. (2015). 2015 Plastics-To-Fuel Project Developers Guide. Ocean Recovery Alliance, (June), 74.

TORRES MORENO, A. K., \& CASTAÑEDA SÁENZ, P. C. (2018). Evaluación De La Obtención De Diésel a Nivel Laboratorio Mediante Pirólisis a Partir De Residuos Plásticos Provenientes Del Proceso De Empacado En La Empresa Atlantic Fs S.a.S. Fundación Universidad de América, 1-152. Retrieved from https://repository.uamerica.edu.co/bitstream/20.500.11839/6951/1/6131986-20182-IQ.pdf

Vitz, E., Moore, J. W., Shorb, J., Prat-Resina, X., Wendorff, T., \& Hahn, A. (2019). Sorting Recyclable Plastics by Density. Chemistry LibreTexts, 2-5. Retrieved from https://chem.libretexts.org/@go/page/50006

Zhang, B., Zhong, Z., Li, T., Xue, Z., Wang, X., \& Ruan, R. (2018). Biofuel production from distillers dried grains with solubles (DDGS) co-fed with waste agricultural plastic mulching films via microwave-assisted catalytic fast pyrolysis using microwave absorbent and hierarchical ZSM-5/MCM-41 catalyst. Journal of Analytical and Applied Pyrolysis, 130(February), 249-255. https://doi.org/10.1016/j.jaap.2018.02.007

Zheng, Y., Tao, L., Yang, X., Huang, Y., Liu, C., \& Zheng, Z. (2018). Study of the thermal behavior, kinetics, and product characterization of biomass and low- 
density polyethylene co-pyrolysis by thermogravimetric analysis and pyrolysisGC/MS. Journal of Analytical and Applied Pyrolysis, 133, 185-197.

https://doi.org/10.1016/j.jaap.2018.04.001 